5 AUSTIN, E. J., and JAROS, M.: 'Electronic structures of an isolated GaAs-GaAlAs quantum well in a strong field', Phys. Rev. B, 1985, B31, p. 5569

6 NOJIMA, S.: 'Enhancement of excitonic electrorefraction by optimizing quantum well materials and structures', Appl. Phys. Lett. 1989,55 , p. 1868

7 NOIMA, S., and WAKITA, K.: 'Optimization of quantum well materials and structures for excitonic electroabsorption effects' Appl. Phys. Lett., 1988, 53, p. 1958

8 KOREN, U., MILLER, B. I., KOCH, T. L., EISENSTEIN, G., TUCKER, R. S BAR-JOSEPH, I., and CHEMLA, D. S.: 'Low-loss InGaAs/Inp multiple quantum well optical electroabsorption waveguide modulato', Appl. Phys. Lett., 1987, 51, p. 1132

\section{K-BAND SUSPENDED SUBSTRATE STRIPLINE TAPPED COMBLINE FILTER}

Indexing terms: Filters, Microwave filters, Circuit theory and design

A newly developed $19 \cdot 5-20 \cdot 5 \mathrm{GHz}$ combline bandpass filte realised by multiple coupled suspended substrate stripline (MCSSSs) and metal-insulator-metal (MIM) capacitors is presented. The filter design uses an iterative field-theorybased algorithm to account for the composite effects of multiple dominant couplings through the nonadjacent and tested. The measured and theoretical filter characteristics are in good agreement for the combline filter without any tuning.

Introduction: Many useful properties ${ }^{1,2}$ have made the combline filter one of the widely used bandpass filters in many communication systems. The tapped-line arrangement makes the combline filter even more compact and easier to interface the input and output ports. ${ }^{3.4}$ Recently, a miniaturised, lowcost, and light-weight tapped combline filter realised by microstrip lines and beam-lead capacitors has been designed and reported. ${ }^{5}$ Using the method described in Reference 5 , $40 \%$ bandwidth combline filter was obtained easily. It showed that, by equalising the propagation constants of the multiple quasi-TEM modes associated with the coupled multiple transmission lines (MTLs), the stopband performance can be substantially improved. To reduce the cost of selecting and using the beam-lead capacitors and to improve the $Q$ factor of the transmission line, a new combline filter using multiple coupled suspended substrate striplines (MCSSSs) is developed and tested successfully. The new arrangement makes the values of MIM capacitors flexible, without being limited by vendors. The assembly cost of the new combline filter is significantly lower than that using the beam-lead capacitors because the MIM capacitors involve only photolithography techniques in the fabrication phase. Furthermore, the use of a field-theorybased approach for designing the MCSSS combline filter eliminates the mechanical tuning required for the conventional metal bars combline filter.

Design of MCSSS combline filter using MIM capacitors: Fig. is a three-dimensional view of the new combline filter configu-

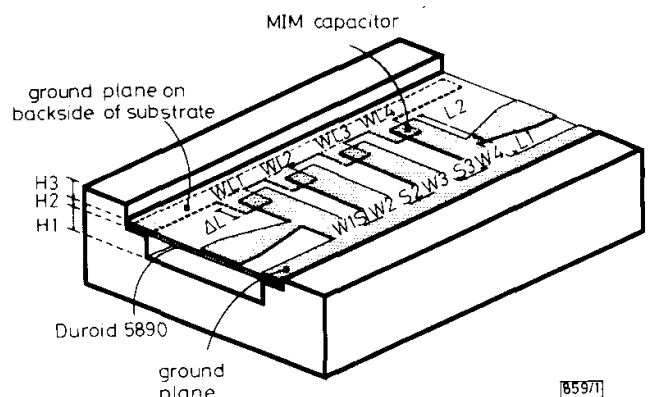

Fig. 1 Three-dimensional view of new combline filter configuratin with top cover taken off ration with the top cover taken off. The input port and output port are tapered from $50 \Omega$ to $75 \Omega$, which is the internal nor malised impedance of the filter. The MCSSSs and the MIM capacitors constitute the resonator sections. One side of the MCSSSs is grounded and the other one is connected by the MIM capacitors, which are also grounded by the opposite side metal strip. The MCSSSs are on top of the 2 mil thick Duroid 5890 substrate. The MIM capacitors, however, are printed on both sides of the substrate. The MCSSS configuration contributes at least two intertwined effects. One is the existence of the multiple dominant quasi-TEM modes. The other is the undesired couplings through nonadjacent MCSSSs. A field-theory-based iterative design approach similar to the one reported in Reference 5 is applied to account for the composite effects of the two undesired physical conditions. The use of field-theory approach enables the physical layout of the new MCSSS combline filter to be tuned to meet the prescribed filter characteristics. This approach is drastically different from the design of conventional combline filter using parallel metal bars, which support only one TEM mode. ${ }^{1-3}$

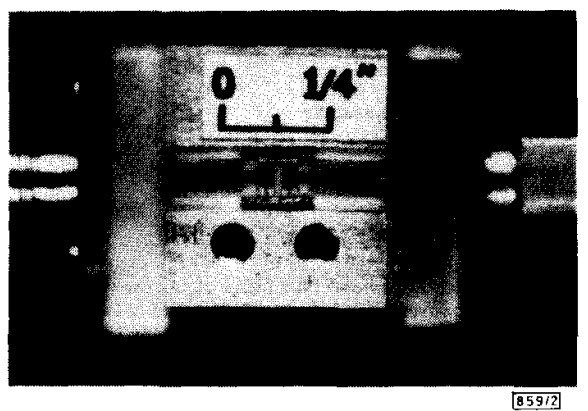

Fig. 2 Photograph of prototype tapped combline filter Structural parameters:

(units in mil)

(units in mil)

$W 1=W 4=W L 1=W L 4=11.45$

$W 2=W 3=W L 2=W L 3=11 \cdot 50$

$\Delta L 1=\Delta L 4=15 \cdot 10, \Delta L 2=\Delta L 3=15 \cdot 00, L 1=36 \cdot 07, L 2=46 \cdot 00$ $S 1=S 3=10 \cdot 40, S 2=21 \cdot 70, H 1=10 \cdot 00, H 2=2 \cdot 00, H 3=30 \cdot 00$

$C 1=C 4=0.055 \mathrm{pF}, C 2=C 3=0.055 \mathrm{pF}$

Substrate: Duroid 5890, $\varepsilon_{\mathrm{p}}=2 \cdot 1$

Theoretical and measured filter characteristics: The photogra$\mathrm{ph}$ of the prototype of the MCSSS combline filter is shown in Fig. 2. It features $14.4 \mathrm{~mm}$ by $2.5 \mathrm{~mm}$ in size including the tapered sections. Also given are the structural parameters, which are commensurate with standard microwave printedcircuit-board photolithography techniques. The Duroid 5890 has a relative permittivity of $2 \cdot 1$. Fig. 3 compares the theoreti cal and measured filter characteristics of insertion loss and

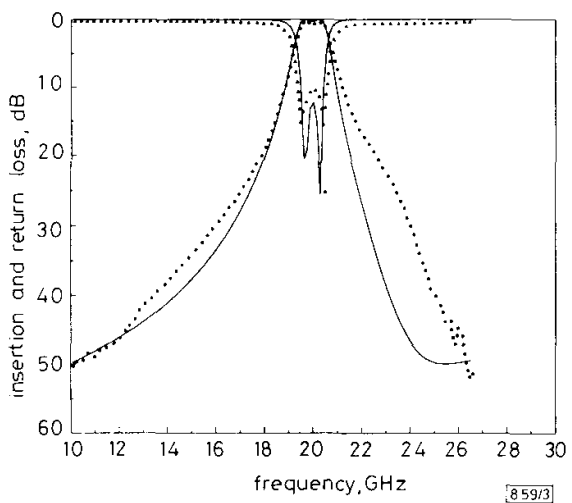

Fig. 3 Theoretical and measured results

measured insertion loss

measured return loss

theoretical results 
return loss. The theoretical results assuming that the metal strips and the electric housing are perfect metal are illustrated by solid lines. The measured insertion loss and return loss are also shown. These plots agree favourably. The measured characteristics show $1 \mathrm{~dB}$ loss in the passband. This is attributed mainly to the finite ground plane conductivity and conductor losses in the MCSSSs.

Conclusion: The MCSSS realisation of a tapped combline bandpass filter using MIM capacitors has been built and tested successfully. The key step in designing such filters without any tuning is the use of an iterative design approach incorporating the field-theory-based analysis of the physical layout of the combline filter. This reduces the cost and labour of implementing MCSSS combline filters. The measured filter characteristics are also in good agreement with the theoretical predictions, which assumed a loss-free conductor for MCSSS and electric housing.

Acknowledgment: This study was supported by the National Science Council, Republic of China, under contract number: NSC 79-0404-E009-29.

W.T. LO

23rd May 199I

C.-K. C. TZUANG

Institute of Communication Engineering

National Chiao Tung University

No. 75, Po-Ai Street

Hsinchu, Taiwan, Republic of China

\section{References}

1 MAITAEI, G. L.: 'Comb-line band-pass filters of narrow or moderate band width', Microwave $J$., 1963, 6, pp. 82-91

2 WENZEL, R. J.: 'Synthesis of combline and capacitively loaded interdigital bandpass filters of arbitrary band width', IEEE Trans., 1971, MTT-19, (8), pp. 678-686

3 CRISTAL, E. G.: 'Tapped-line coupled transmission lines with applications to interdigital and combline filter', IEEE Trans., 1975, MTT-23, pp. 1007-1012

4 wONG, s.: 'Microstrip tapped-line filter design', IEEE Trans., 1979, MTT-27, (1), pp. 44-50

5 TZUANG, C.-K. C., and 10, W.-T.: 'Printed-circuit realization of a tapped combline bandpass filter'. 1990 IEEE MTT-S Int. Symp. Dig., session B-3, pp. 131-134

\section{NOISE PERFORMANCE OF DISTRIBUTED FIBRE AMPLIFIERS}

\author{
Indexing terms: Amplifiers, Noise, Optical fibres
}

Noise measurements on a distributed erbium-doped fibre amplifier are reported for the first time. Data are presented for fibre lengths up to $54 \mathrm{~km}$, and are in good agreement with theoretical predictions.

Introduction: Distributed optical amplification has been demonstrated over tens of kilometres using low concentration erbium-doped fibre and modest $1480 \mathrm{~m}$ pump powers. ${ }^{1}$ Potential applications which may require low signal power excursions include nonlinear transmission systems, and lossless distribution networks. Noise will be an important consideration for any transmission system application, and in this Letter we consider the noise performance of distributed amplifiers. Theoretical noise figure predictions are contrasted with conventional 'lumped' amplification, and supporting experimental data are presented.

Optical amplifier noise figure: Optical amplification is accompanied by the addition of amplified spontaneous emission (ASE), which is quantified by the amplifier noise figure, defined as the signal to noise ratio degradation from the input to output of the amplifier. Optical amplifier noise figures can be expressed as ${ }^{2}$

$$
N F=2 F+G^{-1}
$$

where $G$ is the amplifier gain. $F$ is the equivalent input noise photon flux per unit frequency, and represents the 'excess noise factor'. A noise figure of $3 \mathbf{d B}$ is the fundamental limit for high gain, phase-insensitive optical amplification. ${ }^{2,3}$

It is appropriate to compare the noise performance of a distributed amplifier with lumped amplification over an equivalent fibre span. Three options are illustrated schematically in Table 1: a 'power amplifier', where the lumped amplification precedes the fibre span, a distributed amplifier with uniform gain or loss along the length of the fibre, and a 'preamplifier', where the lumped amplification follows the fibre span. In all three cases the overall net linear gain is $G$, and linear fibre loss is $L(L>1)$. (For the distributed amplifier, $L$ is the background loss of the fibre.)

Table 1 OPTICAL AMPLIFICATION OPTIONS FOR LINK WITH OVERALL LINEAR GAIN OF $G$, OVER FIBRE SPAN WITH LINEAR LOSS $L(L>1)$

\begin{tabular}{|c|c|c|c|}
\hline \multirow{2}{*}{ Option } & \multirow[t]{2}{*}{ Schematic diagram } & \multicolumn{2}{|c|}{ Excess noise factor } \\
\hline & & General & $G=1$ \\
\hline $\begin{array}{l}\text { Power } \\
\text { amplifier }\end{array}$ & $\overbrace{\substack{\text { amplifier } \\
\text { gain } G L}}^{(0)}$ & $\frac{G L-1}{G L}$ & $\frac{L=1}{L}$ \\
\hline $\begin{array}{l}\text { Distributed } \\
\text { amplifier }\end{array}$ & $\frac{(Q)}{\begin{array}{c}\text { fibre span background } \\
\text { loss } L, \text { net gain } G\end{array}}$ & $\frac{\ln (G L)}{\ln G} \frac{G-1}{G}$ & $\ln L$ \\
\hline Preamplifier & $\underset{\substack{\text { fibre span } \\
\text { loss } L}}{\substack{\text { amplifier } \\
\text { gain } G L}}$ & $\frac{G L-1}{G}$ & $L-1$ \\
\hline
\end{tabular}

Excess noise factor formulas assume uniform spontaneous emission factor of one and scale linearly for larger values

It can readily be argued that the noise performance of the distributed amplifier must lie between the two extremes of the power amplifier and preamplifier, ${ }^{4}$ and Table 1 lists the excess noise factors for the three amplifier options. The formulas assume complete population inversion to give a spontaneous emission factor of one, ${ }^{3}$ and scale linearly for larger values. For the power amplifier and preamplifier configurations, $F$ is given by conventional lumped amplifier noise theory, but the distributed amplifier calculation must include the background fibre loss. One approach is to determine the noise performance of a link with periodic discrete amplification in the

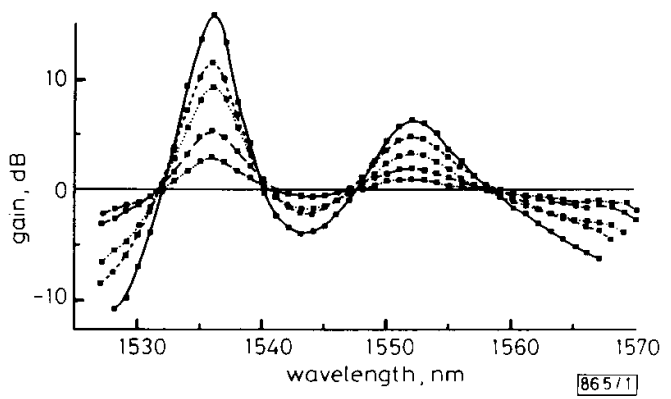

Fig. 1 Distributed erbium doped fibre amplifier gain spectra

Zero net gain at $1532,1540,1547.5$ and $1558.5 \mathrm{~nm}$

- $53.5 \mathrm{~km}$

$-3.5 \mathrm{~km}$
$-0.5 \mathrm{~km}$

$-37.5 \mathrm{~km}$
$-18.5 \mathrm{~km}$

$-18.5 \mathrm{~km}$

... $10 \mathrm{~km}$

ELECTRONICS LETTERS 18th JulY 1991 Vol. 27 No. 15 